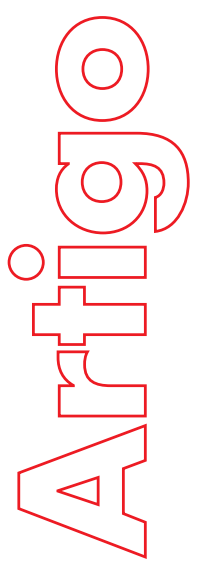

\title{
ESTUDO SOBRE INFRAESTRUTURA DE DADOS ESPACIAIS PARA EMBASAR A PROPOSTA DE DESENVOLVIMENTO DE UMA IDE PARA A UNIVERSIDADE FEDERAL DE VIÇOSA
}

\author{
Priscila de Lima e Silva \\ Afonso de Paula dos Santos \\ Jugurta Lisboa Filho
}

Revista

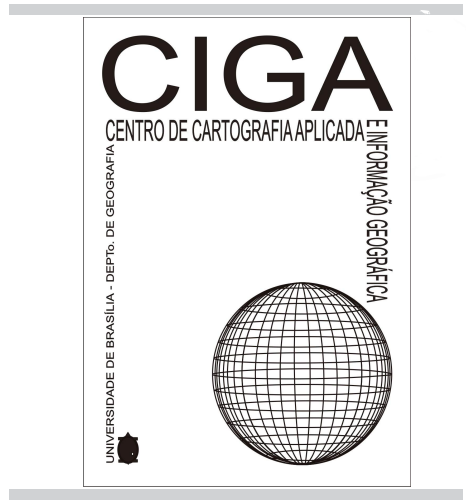

Revista Eletrônica:

Tempo - Técnica - Território,

V.9, N.2 (2018), 07:27

ISSN: $2177-4366$ p. $07-27$

Como citar este ar tigo:

Silva, P.L.; Santos. A.P.; Filho , J.L.; CARTOGRAFIA DO IMPEACHMENT: REPRESENTAÇÃO ESPACIAL DO PROCESSO POLÍTICO NO BRASIL EM 2016-2017. Revista Eletrônica: Tempo - Técnica - Território, v.9, n.2 (2018), p. 07:27

ISSN: 2177-4366.

\section{Disponível em:}

http://periodicos.unb.br/index.php/ciga/issue/view/1905

Este obra está licenciado com uma Licença Creative Commons Atribuição - Não Comercial 4.0 Internacional. 


\title{
ESTUDO SOBRE INFRAESTRUTURA DE DADOS ESPACIAIS PARA EMBASAR A PROPOSTA DE DESENVOLVIMENTO DE UMA IDE PARA A UNIVERSIDADE FEDERAL DE VIÇOSA
}

\author{
Priscila de Lima e Silva \\ Universidade Federal de Viçosa \\ Departamento de Engenharia Civil \\ Mestranda em Engenharia Civil/ Informações Espaciais \\ Engenheira Agrimensora e Cartógrafa \\ priscila.l.silva@ufv.br \\ Afonso de Paula dos Santos \\ Universidade Federal de Viçosa \\ Departamento de Engenharia Civil \\ Setor de Engenharia de Agrimensura e Cartográfica \\ Professor Adjunto \\ Doutor em Informações Espaciais \\ Mestre em Informações Espaciais \\ Engenheiro Agrimensor \\ afonso.santos@ufv.br \\ Jugurta Lisboa Filho \\ Universidade Federal de Viçosa \\ Departamento de Informática \\ Professor Titular \\ Doutor em Ciência da Computação \\ Mestre em Engenharia de Sistemas e Computação \\ Bacharel em Informática \\ jugurta@ufv.br
}


RESUMO: Uma Infraestrutura de Dados Espaciais (IDE) é uma plataforma que permite interligar produtores e usuários de informações espaciais. Consolidada sobre cinco principais componentes: dados, pessoas, tecnologias, padrões e políticas; uma IDE possibilita interoperabilidade no compartilhamento e divulgação de informações geográficas. Desta forma, proporciona redução de custos na produção de informações espaciais, evitando que estes dados sejam produzidos de forma duplicada e facilita a utilização das informações, deixando-as disponíveis de forma padronizada. Diversos países produziram IDEs Nacionais, como é o caso do Brasil, onde através no Decreto 6.666 foi implementada a Infraestrutura Nacional de Dados Espaciais - INDE. Este decreto prevê que outras camadas da hierarquia governamental também atuem no desenvolvimento de infraestruturas para disponibilização dos dados espaciais por eles produzidos. Neste sentido, este artigo apresenta uma revisão dos conceitos sobre IDE, discutindo as IDE já desenvolvidas no Brasil, e apresentado a proposta de desenvolvimento de uma IDE Universitária para a Universidade Federal de Viçosa (UFV). Na universidade é grande a produção de informações geográficas, devido aos projetos de pesquisa, ensino e extensão, bem como a demanda para a administração territorial do campus. Além disto, grande parte destes projetos são custeados por verbas públicas, o que mostra a importância da criação de infraestruturas que disponibilizem estes dados, para evitar custos em produção duplicada e organizar, através de padrões, as informações existentes. Uma preocupação existente, quanto a execução deste projeto, é garantir que existam mecanismos para atualização e continuidade da IDE dentro da universidade. Desta forma, junto a Pró-Reitoria de Administração da UFV, estão sendo discutidas formas de institucionalizar a IDE na universidade.

Palavras-chave: Infraestrutura de dados Espaciais (IDE), Informação Espacial, Universidade

ABSTRACT: A Spatial Data Infrastructure (IDE) is a platform that allows the interconnect of users and spatial information. Consolidated on five main components: data, people, technologies, standards and policies; an IDE enables interoperability in sharing and dissemination of geographic information. In this way, provides reduced costs in the production of spatial data, avoiding that this data is produced in duplicate form and facilitates the use of the information, making it available in a standardized way. Several countries have produced National IDEs, as is the case in Brazil, where by Decree 6,666 the National Spatial Data Infrastructure - INDE was implemented. This decree foresees that other layers of the governmental hierarchy also act at the development of infrastructures for the availability of spatial data produced by them. In this sense, this article presents a review of the concepts about IDE and presents a proposal for the development of a University IDE for the Federal University of Viçosa (UFV). At university it's large the production of geographic information, in research projects, teaching and extension, as well as a demand for a campus territorial administration. In addition, a large part of these projects are funded by public funds, which shows the importance of creating the infrastructures that make this data available, for to avoid costs in duplicate production and to organize, through standards, the existing information. An existing concern about the execution of this project is there are mechanisms for updating and maintaining IDE within the university. Thus, together with the Administration of UFV, ways of institutionalizing an IDE in the university are being discussed.

Key words: Spatial Data Infrastructure (IDE), Spatial Information, University

RESUMEN: Una Infraestructura de Datos Espaciales (IDE) es una plataforma que permite interconectar productores y usuarios de informaciones espaciales. Consolidada sobre cinco componentes principales -datos, personas, tecnologías, estándares y políticas- una IDE hace posible 
la interoperabilidad a la hora de compartir y divulgar informaciones geográficas. De esta forma, proporciona una reducción de costes en la producción de informaciones espaciales, evitando que estos datos se produzcan de forma duplicada, y permite una mejor utilización de las informaciones existentes. Varios países produjeron IDES nacionales, como Brasil, donde a través del Decreto 6.666 se implementó la Infraestructura Nacional de Datos Espaciales -INDE. Este decreto prevé que otras capas de la jerarquía gubernamental también se envuelvan en el desarrollo de infraestructuras para tornar disponibles datos espaciales por ellas producidos. En este contexto, se presenta la propuesta de desarrollo de una IDE universitaria para la Universidad Federal de Viçosa (UFV). En esta universidad es grande la producción de información geográfica debido a los proyectos de investigación, enseñanza y extensión y la demanda para la administración territorial del campus. Asimismo, gran parte de estos proyectos son costeados por dinero público, lo que muestra la importancia de crear infraestructuras que aseguren la disponibilidad de estos datos para evitar costes de producción duplicados y organizar, a través de estándares, la información existente. Una preocupación presente, en lo que respecta a la ejecución de este proyecto, es garantizar que existan mecanismos para la actualización y continuidad de la IDE dentro de la universidad. De esta forma, junto con la Prorrectoría de Administración de la UFV, se están discutiendo formas de institucionalizar la IDE en la universidad.

Palabras clave: Infraestructura de Datos Espaciales (IDE), información espacial, universidad

\section{Introdução}

Atualmente vivemos na era da informação, onde as informações geográficas têm grande importância para a tomada de decisões em diversos setores. Como o mostrado por Janssen (2011) o setor público recolhe e utiliza dados espaciais para preparar, implementar, avaliar políticas e prestar serviços eficientes aos cidadãos. Já o setor privado necessita de dados espaciais confiáveis para desenvolver produtos ou serviços de informação, tais como sistemas de navegação, relatórios meteorológicos ou serviços imobiliários.

A obtenção de dados espaciais requer tempo e recursos financeiros, assim o que muitas organizações almejam é uma maneira de facilitar o acesso as informações espaciais já geradas, de maneira a evitar custos para produção duplicada de informações (RAJABIFARD \& WILLIAMSON, 2001).

A partir do entendimento dos benefícios possíveis a partir do compartilhamento de informações geográficas, surgiu o conceito de Infraestrutura de Dados Espaciais (IDE), que pode ser vista como uma plataforma que interliga os produtores de dados, os fornecedores e os agregadores de valor aos utilizadores dos dados (RAJABIFARD \& WILIAMSON, 2005).

A IDE pode ser definida como uma plataforma habilitadora para compartilhamento de dados, que é baseada em um conceito hierárquico e multidisciplinar. Uma IDE inclui pessoas, dados, redes de acesso, políticas, normas técnicas e recursos humanos; visando facilitar e coordenar o intercâmbio de dados espaciais entre as partes interessadas (RAJABIFARD et al., 2006).

Mais que armazenamento de dados, a IDE é uma hospedagem de dados espaciais e seus atributos, suficientemente documentados, disponibilizados para avaliação, consulta e exploração, 
possuindo suas informações padronizadas, respeitando os acordos governamentais e organizacionais (GSDI, 2004).

No Brasil, em novembro de 2008 foi instituída a Infraestrutura Nacional de Dados Espaciais (INDE) através do Decreto $\mathrm{n}^{\circ}$ 6.666. A INDE tem como objetivo promover o ordenamento na geração, armazenamento, compartilhamento, disseminação e uso dos dados espaciais; além de promover a utilização de normas homologadas pela Comissão Nacional de Cartografia (CONCAR) e evitar duplicidade na obtenção de dados espaciais pelos órgãos públicos (BRASIL, 2008).

Através do Decreto $\mathrm{n}^{\circ} 6.666$ foi definido a obrigatoriedade de disseminação dos dados espaciais e metadados referentes a estes dados por todos os órgãos e entidades públicas federais (BRASIL, 2008). A INDE permite que produtores de informações espaciais, como entidades e empresas, atuem ativamente no compartilhamento dessas informações tornando-se um nó externo provedor ou produtor de informações espaciais da infraestrutura nacional (BRASIL, 2010).

As universidades públicas têm se mostrado grandes produtoras e utilizadoras de informações espaciais. Pois nos diversos projetos de pesquisa, ensino e extensão são produzidos e analisados dados geográficos. Além disto, para a uma boa administração territorial dos campi universitários se faz necessário o conhecimento do espaço, sendo este caracterizado por dados cartográficos.

Desta forma, as universidades são órgãos públicos que necessitam de infraestruturas para o compartilhamento de informações espaciais. A partir de uma IDE Universitária é possível permitir a melhor utilização das informações geográficas, reduzir os custos para a produção destas informações e cumprir com o disposto no Decreto 6.666, quanto a obrigatoriedade de divulgação dos dados cartográficos produzidos por instituições públicas.

Este trabalho tem como objetivo apresentar uma revisão bibliográfica sobre IDE. Esta revisão foi elaborada para embasar a proposta de desenvolvimento de uma IDE Universitária para a Universidade Federal de Viçosa (UFV). A proposta concebida é apresentada nas considerações finais, tais como as medidas em andamento para a concretização desta.

\section{Desenvolvimento}

\subsection{Evolução das Infraestruturas de Dados Espaciais}

Um fator que impulsionou a evolução das IDEs no mundo foi o aumento da conscientização sobre a importância do compartilhamento de bases de dados geoespaciais, com ênfase à integração, compatibilização e disponibilização daquelas informações consideradas de uso comum. Os acordos para promover uma IDE inicialmente foram feitos entre órgãos públicos e atualmente abrangem todos os atores da sociedade (BRASIL, 2010). 
A evolução das IDEs é dividida em três gerações, como mostra a Figura 1. De acordo com Craglia et al. (2008) a primeira geração de IDE foi em grande parte liderada por agências nacionais e orientada para a geração de bases de dados espaciais nacionais que abordavam o mapeamento topográfico e camadas de uso geral. Na primeira geração de IDE, os dados foram o principal propulsor do desenvolvimento de iniciativas (RAJABIFARD et al., 2006).

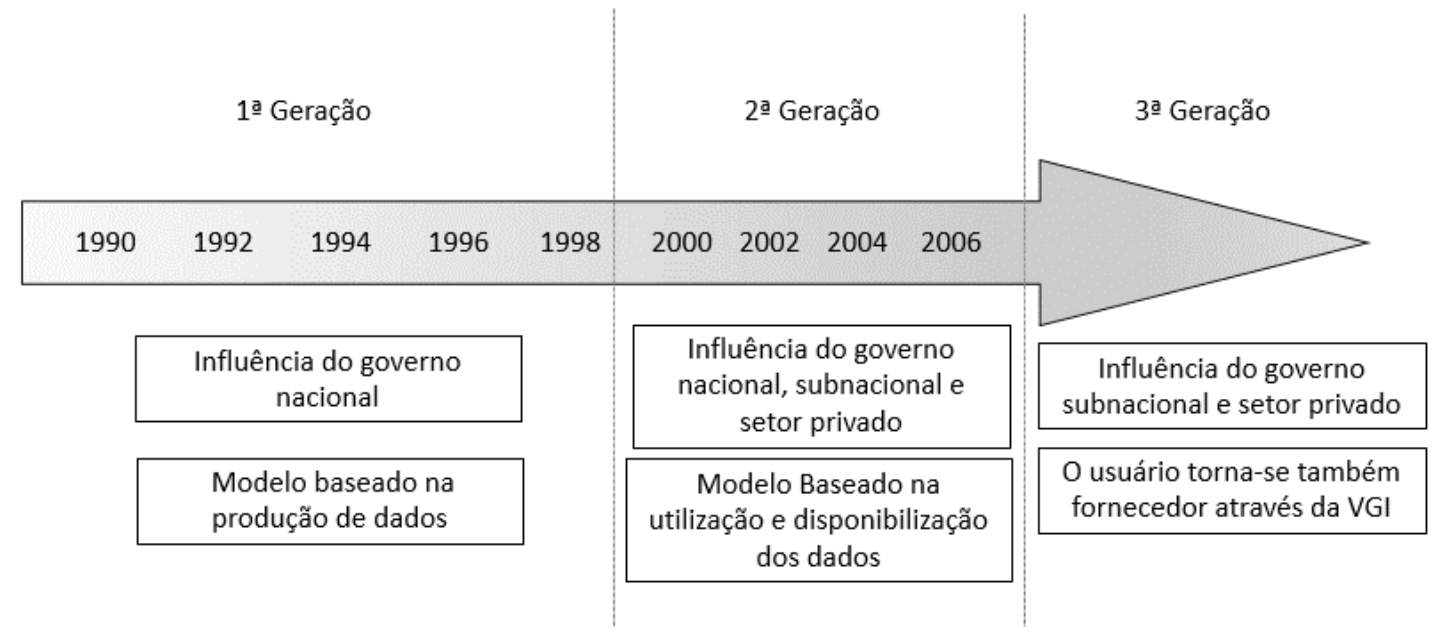

Figura 1 - Evolução da IDE

Fonte: Adaptado de RAJABIFARD et al. (2006)

A segunda geração foi dirigida pelas necessidades dos usuários, com foco sobre a utilização e aplicação dos dados em oposição aos dados em si. Nessa geração foi incluída a introdução aos serviços web de partilha e comunicação de dados, bem como impulsionado a publicação e difusão dos dados desenvolvidos na primeira geração (RAJABIFARD et al., 2006).

Já a terceira e atual geração, de acordo com Rajabifard et al. (2006), tem como principal diferença o fato de que as IDEs desenvolvidas não são predominantemente as nacionais, provindas do serviço público. Assim, é crescente o surgimento de IDEs regionais e a atuação do poder privado. Já outros autores, como Budhathoki et al. (2008), acreditam que o diferencial da terceira geração é a inclusão de informações geográficas voluntárias (VGI), onde os usuários nesta nova geração, também atuam como fornecedores de informação.

\subsection{Classificação dos tipos de IDEs}

Rajabifard et al. (2000) apresenta que uma IDE pode ser classificada de forma hierárquica, onde no topo estão as IDEs globais e regionais, que abrangem grande área territorial e na base estão localizadas as IDEs locais e coorporativas, que abrangem menores áreas, mas representam um maior nível de detalhamento, conforme apresentado na Figura 2.

O relacionamento entre IDEs pode ocorrer em nível vertical onde uma IDE de classe superior pode ser utilizada para o desenvolvimento de uma IDE de classe inferior, podendo também ocorrer o contrário. Existe também, interação em nível horizontal, onde IDEs de mesma categoria podem ser Revista Eletrônica: Tempo - Técnica - Território, V.9, N.2 (2018), 7:27 ISSN: 2177-4366 
associadas. No entanto, para realizar essas interações existe a preocupação com a interoperabilidade entre os dados existentes nas diferentes IDEs (RAJABIFARD et. al., 2000).

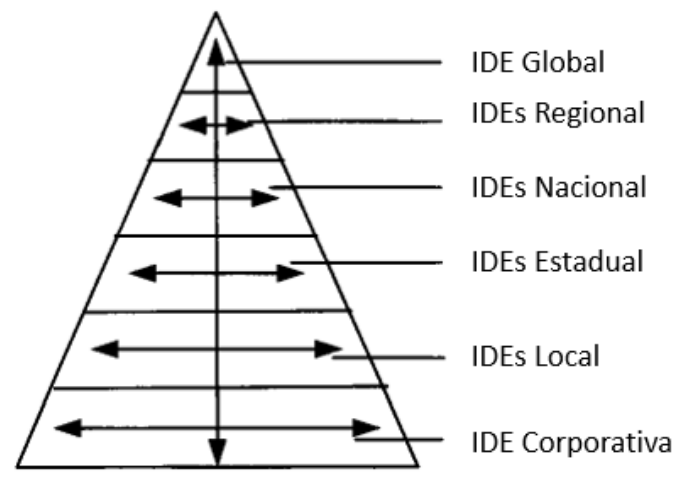

Figura 2 - Hierarquia de uma IDE Fonte: Traduzido de RAJABIFARD et. al. (2000)

\subsection{Objetivo de uma IDE}

De acordo com Rajabifard et al. (2006) uma IDE é desenvolvida com o objetivo de apoiar o acesso imediato à informação espacial e a tomada de decisões, em diferentes escalas e para múltiplos propósitos. O que permite que os usuários economizem recursos, tempo e esforço para adquirir novos dados, evitando duplicação de despesas relacionadas a geração e manutenção de dados.

Já Masser (2002) destaca os seguintes objetivos de uma IDE:

- Compartilhar informação geográfica, inicialmente para a administração pública e posteriormente para toda a sociedade;

- Garantir aos cidadãos acesso a informação geográfica pública;

- Incorporar a informação geográfica produzida pela iniciativa privada;

- Harmonizar a informação geográfica disponibilizada e registrar as características dessa informação;

- Subsidiar a tomada de decisões de forma mais eficiente e eficaz.

\subsection{Justificativa para implementação de uma IDE}

A produção de dados geográficos é muito importante para a gestão e planejamento territorial, mas não basta produzir, é preciso permitir e facilitar o uso. A informação espacial é uma mercadoria que só tem valor uma vez que circule e, com isso, alavanque mais valor (BORBA et al., 2014).

Borba et al. (2014) apresenta os seguintes fatores que tem dificultado a reutilização de dados espaciais:

- Produção descentralizada com métodos diferenciados;

- Documentação rudimentar sobre a metodologia e padrão utilizados na produção; 
- Dificuldades burocráticas de acesso aos dados;

- Desconhecimento dos acervos existentes.

Uma IDE soluciona os problemas de reutilização de dados espaciais, pois, como o apresentado por Nakamura (2010), uma infraestrutura de dados espaciais proporciona instrumentos técnicos e políticos que ampliam o ciclo de uso das informações geográficas para um maior número de pessoas, visando suprir problemas de interoperabilidade, compartilhamento e padronização de dados.

Nedovic-Budic e Pinto (2000) apresentam os seguintes benefícios ao se realizar o compartilhamento de dados espaciais:

- Economia de custos - através da partilha dados, já não existe a necessidade de duplicar a recolha e o arquivamento de dados, o que conduz a economias em termos de pessoas, espaço, instalações, aquisição de dados e custos para produção e manutenção;

- Maior disponibilidade de dados - o banco de dados de uma IDE possui informações espaciais de diversas fontes, portanto é mais completo que um banco de dados de uma única organização, oferecendo assim uma biblioteca mais abrangente de informações geográficas;

- Relações interorganizacionais melhoradas - através da partilha de dados ocorre a promoção de uma maior comunicação interorganizacional, assim supõe-se, que entre as organizações que comunicam e compartilham informações, exista uma oportunidade mais forte para o desenvolvimento de novas missões de serviço em conjunto dentro de suas áreas de atuação. No entanto, para concretizar as vantagens de uma IDE e acelerar o seu desenvolvimento, Rajabifard e Wiliamson (2001) apontam alguns fatores que devem ser considerados:

- Conscientização do uso da Informação Geográfica e IDEs;

- Cooperação entre as várias partes interessadas;

- Envolvimento dos políticos envolvidos;

- Conhecimento sobre o tipo, localização, qualidade e propriedade dos conjuntos de dados;

- Acessibilidade aos conjuntos de dados;

- O uso bem-sucedido e generalizado dos conjuntos de dados.

Desta forma uma IDE é um mecanismo que facilita o compartilhamento e utilização de dados espaciais. No entanto, para que uma IDE alcance todo seu potencial, além das questões técnicas (como os dados fornecidos, a qualidade destes dados e os softwares empregados), questões políticas devem ser consideradas, para que exista apoio para a construção, manutenção e difusão da infraestrutura.

A importância da IDE se traduz pelo potencial de compartilhamento das informações geográficas, integrando diversos tipos de usuários, diminuindo a falta de interoperabilidade entre diferentes sistemas e criando uma padronização dos dados. É um conceito simples, mas existe uma 
dificuldade de implantação devida as diferenças tecnológicas, políticas e culturais dos produtores de dados geográficos (NAKAMURA e FILHO, 2012).

A Figura 3 mostra como o desenvolvimento de uma IDE pode melhorar o compartilhamento das informações geográficas. Antes do desenvolvimento de IDEs somente os profissionais da área possuíam amplo acesso aos dados espaciais, estes realizavam estudos e transmitiam os resultados aos cidadãos e aos órgãos públicos na forma impressa, por exemplo. Com o desenvolvimento de IDEs além de acesso aos resultados, os cidadãos e órgãos públicos possuem acesso as informações espaciais, assim eles também podem desenvolver análises e obterem resultados que necessitam.
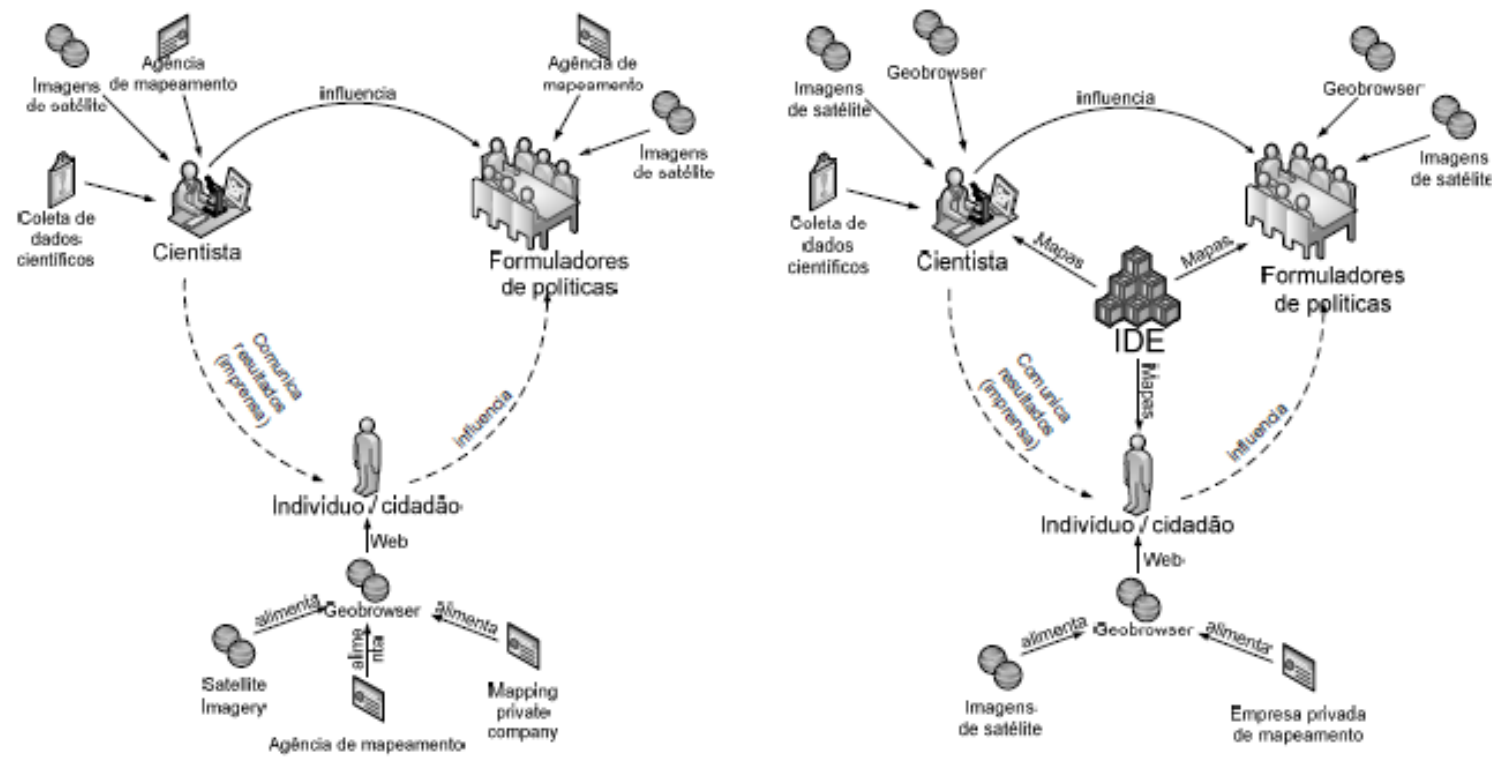

Figura 3 - Fluxo de Informações Espaciais antes e após o desenvolvimento de uma IDE Fonte: DAVIS et al., 2009

\subsection{Componentes de uma IDE}

Uma IDE vai além dos dados, fornece um ambiente no qual organizações e/ou nações interagem com tecnologias para promover atividades para o uso, gerenciamento e produção de dados geográficos (RAJABIFARD \& WILIAMSON, 2001). Assim de acordo com Warnest (2005) é possível definir cinco componentes para uma IDE:

Dados: constituem o componente central de uma IDE, podem ser compreendidos como os vários conjuntos de dados geoespaciais, como dados de controle geodésico, cadastro, limites administrativos, hidrografia, entre outros. Os dados dependem da finalidade e dos órgãos responsáveis, assim variam para cada IDE.

Pessoas: abrange todas as partes interessadas, os usuários e os produtores de dados, podendo englobar o setor público, privado, acadêmico e a sociedade como um todo. 
Estrutura Institucional: inclui os componentes da administração, coordenação, política e legislação de uma IDE. Através das políticas e dos componentes organizacionais que é possível definir os objetivos e metas da IDE e qual a forma de alcança-los (ONAH, 2009).

Tecnologia: consiste nas redes de acesso e distribuição das informações, como geoportais, que permitem busca, consulta, integração, acesso e utilização dos dados geográficos.

Padrões: os padrões são necessários para habilitar o compartilhamento, integração e distribuição dos dados espaciais, através dos padrões são definidos os modelos de dados e metadados, assim é possível garantir a interoperabilidade das aplicações.

Os componentes de uma IDE estão fortemente relacionados, podendo muitas vezes se sobreporem, existindo relações dinâmicas entre eles, onde as pessoas se conectam aos dados, através das tecnologias, políticas e padrões, como mostra a Figura 4. A natureza dinâmica da relação entre os componentes pode ser explicada pelo avanço constante das tecnologias e as mudanças que ocorrem nas comunidades e nas necessidades dos usuários (RAJABIFARD et al., 2002).

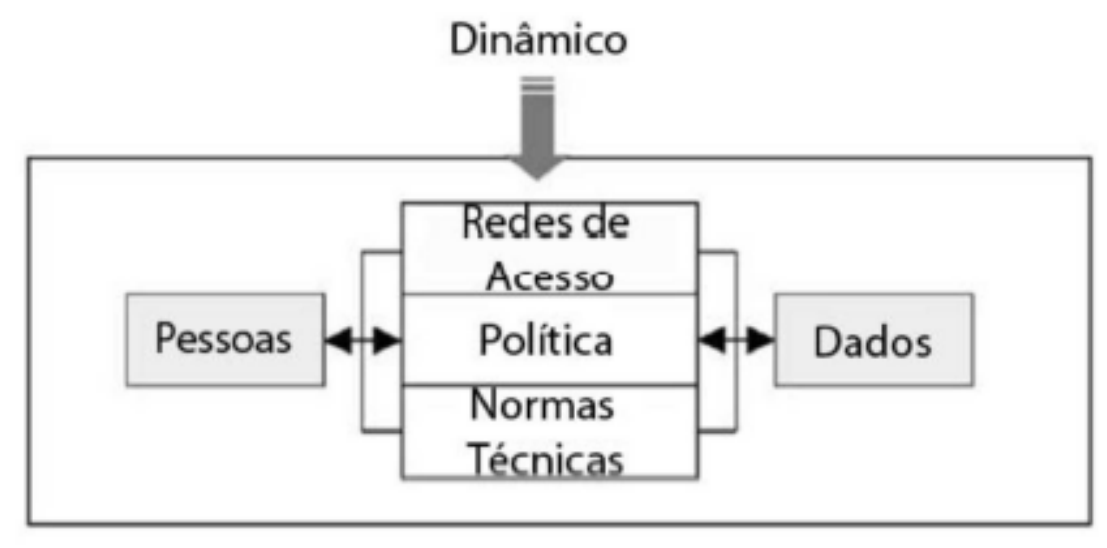

Figura 4 - Interação entre os componentes de um IDE

Fonte: RAJABIFARD \& WILIAMSON (2001)

Alguns autores, como ONAH (2009) e GSDI (2004), além dos componentes descritos, definem também os metadados como um componente das IDEs. Metadados são informações sobre um determinado dado, o Decreto $n^{\circ}$ 6.666/08 define os metadados para informações geoespaciais como

"Conjunto de informações descritivas sobre os dados, incluindo as características do seu levantamento, produção, qualidade e estrutura de armazenamento, essenciais para promover a sua documentação, integração e disponibilização, bem como possibilitar a sua busca e exploração".

O uso efetivo dos dados espaciais é inibido devido à falta de conhecimento da existência dos mesmos, informações mal documentadas e/ou inconsistências. Além disso, os dados espaciais têm uma natureza dinâmica, podem ser destinados a diversos fins. Por estes aspectos, os metadados é um requisito essencial para localizar, avaliar e permitir o uso efetivo dos dados disponíveis. (GSDI, 2004) 


\subsection{Arquitetura computacional}

Um dos principais objetivos de uma IDE é o compartilhamento de dados espaciais, no entanto estes dados são heterogêneos, por serem provenientes de diversas bases de dados, se encontrarem em formatos distintos, com escalas e precisões diferentes. Assim a arquitetura computacional empregada no desenvolvimento de uma IDE tem de ser flexível e aberta, para integrar os dados.

A Arquitetura Orientada a Serviços, ou em inglês Service Oriented Architeture (SOA), é um modelo desenvolvido para organizar e utilizar recursos distribuídos que podem estar sob o controle de diferentes domínios de propriedade (OASIS, 2006). Essa arquitetura tem sido muito utilizada no desenvolvimento de IDEs, por se basear em serviços, é independente de tecnologias, assim consegue facilitar a integração de serviços entre vários sistemas, permitindo o desenvolvimento de uma IDE com interoperabilidade (CARVALHO, 2013).

De acordo com Davis e Alves (2005) a SOA é composta por:

- Serviços: constituem a base da SOA, os serviços são autocontidos, ou seja, as informações que descrevem os serviços podem ser obtidas do próprio serviço, através de um conjunto padronizado de métodos. Podem ser serviços primários, quando são independes de outros serviços, ou compostos, quando parte do seu processamento depende de outros serviços.

- Repositório: local onde os serviços devem ser listados, para que estes possam ser descobertos.

- Provedores: agentes que implementam e publicam os serviços.

- Usuários: agentes que buscam e encontram os serviços através de seus registros, e os requerem aos provedores, os usuários podem ser seres humanos ou clientes de softwares.

Em uma IDE baseada na arquitetura orientada a serviços, os provedores cadastram as informações dos dados e serviços geográficos disponibilizados em um repositório de metadados e os usuários efetuam buscas a este repositório para avaliarem quais serviços e informações atendem suas necessidades. Como mostra a Figura 5, um usuário humano realiza as buscas através de um geoportal, que pode ser entendido como uma página na web o qual permite acesso a informações geográficas. Normalmente um geoportal é dotado de um webmap e apresenta ferramentas de busca e interação com os dados disponibilizados. Já um usuário software-cliente realiza a busca através de um serviço Web de catálogo (DAVIS \& ALVES, 2005). 


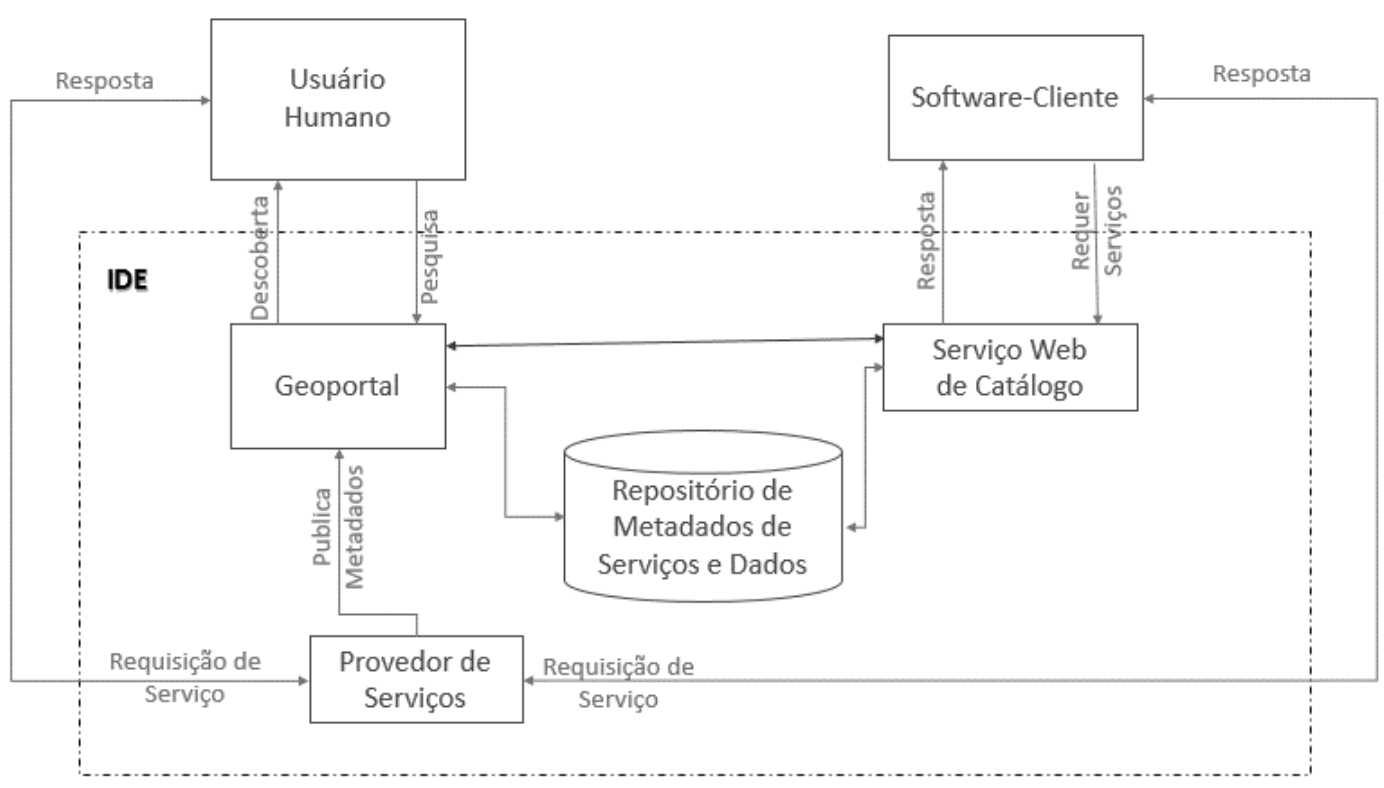

Figura 5 - IDE baseada em SOA

Fonte: DAVIS \& ALVES (2005)

\subsection{Infraestrutura Nacional de Dados Espaciais (INDE)}

O marco legal para o desenvolvimento da Infraestrutura Nacional de Dados Espaciais (INDE) brasileira é o Decreto Presidencial n 6.666 de 27 de outubro de 2008, onde INDE é definida como

"conjunto integrado de tecnologias; políticas; mecanismos e procedimentos de coordenação e monitoramento; padrões e acordos, necessário para facilitar e ordenar a geração, o armazenamento, o acesso, o compartilhamento, a disseminação e o uso dos dados geoespaciais de origem federal, estadual, distrital e municipal”.

Pela definição adotada para INDE brasileira pode-se perceber que o foco desta é no usuário, pois busca unir tecnologias e políticas para desenvolver uma IDE para difundir o uso dos dados espaciais desenvolvidos pelo os órgãos públicos e ordenar o desenvolvimento destes dados a fim de evitar duplicidade.

O Decreto 6.666/08 define também os objetivos da INDE sendo:

- promover o adequado ordenamento na geração, armazenamento, acesso, compartilhamento, disseminação e uso dos dados geoespaciais de origem federal, estadual, distrital e municipal, em proveito do desenvolvimento do País;

- promover a utilização dos padrões e normas homologados pela Comissão Nacional de Cartografia (CONCAR), na produção dos dados geoespaciais pelos órgãos públicos das esferas federal, estadual, distrital e municipal. 
- evitar a duplicidade de ações e o desperdício de recursos na obtenção de dados geoespaciais pelos órgãos da administração pública, por meio da divulgação dos metadados relativos a esses dados disponíveis nas entidades e nos órgãos públicos das esferas federal, estadual, distrital e municipal.

Um dos pilares de uma IDE são as pessoas, para INDE brasileira foram definidos os seguintes atores: instituições governamentais, de todos os níveis do governo; academia, composta por universidades, institutos e centros de pesquisa; iniciativa privada, empresas constituídas com finalidade de lucro; e sociedade, composta pelos cidadãos e sociedade civil organizada (BRASIL, 2010).

Os atores organizacionais e administrativos da INDE foram definidos pelo Decreto 6.666/08 sendo a Comissão Nacional de Cartografia (CONCAR), responsável por coordenar a implantação da INDE de acordo com o plano de ação elaborado, o Instituto Brasileiro de Geografia e Estatística (IBGE), responsável pelas questões técnicas e operacionais da INDE, e o Ministério do Planejamento, Orçamento e Gestão (MP), responsável pelos acordos e cooperações, para o compartilhamento dos acervos de dados geoespaciais.

Um perfil de metadados contém um conjunto básico e necessário de elementos para retratar as características dos produtos geoespaciais de uma determinada comunidade e garantir sua identificação, avaliação e utilização de forma consistente. Assim, a CONCAR criou o Perfil de Metadados Geoespaciais do Brasil (Perfil MGB), baseado na norma ISO 19115/2003 que define um padrão universal para o armazenamento e distribuição de metadados geoespaciais. (CONCAR, 2009)

O Perfil MGB é o que deve ser seguido para confecção dos metadados dos dados a serem armazenados e disponibilizados pela INDE. O Quadro 1 apresenta os elementos do Perfil MGB Sumarizado e a obrigatoriedade definida para estes elementos.

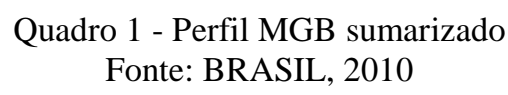

\begin{tabular}{|c|c|c|c|}
\hline \multicolumn{4}{|c|}{ Entidades e elementos do Núcleo de Metadados do Perfil MGB Sumarizado } \\
\hline Entidade / Elemento & Obrigatoriedade & Entidade / Elemento & Obrigatoriedade \\
\hline 1. Título & obrigatório & $\begin{array}{l}\text { 12.Tipo de Representaçã̃o } \\
\text { Espacial }\end{array}$ & opcional \\
\hline 2. Data & obrigatório & 13.Sistema de Referência & obrigatório \\
\hline 3. Responsável & obrigatório & 14.Linhagem & opcional \\
\hline 4. Extensão Geográfica & condicional & 15.Acesso Online & opcional \\
\hline 5. Tdioma & obrigatório & 16.Tdentificador Metadados & opcional \\
\hline $\begin{array}{l}\text { 6. Código de Caracteres do } \\
\text { CDG }\end{array}$ & condicional & $\begin{array}{l}\text { 17. Nome Padråo de } \\
\text { Metadados }\end{array}$ & opcional \\
\hline 7. Categoria Temática & obrigatório & $\begin{array}{l}\text { 18.Versão da Norma de } \\
\text { Metadados }\end{array}$ & opcional \\
\hline 8. Resoluçåo Espacial & opcional & 19.Idioma dos Metadados & condicional \\
\hline 9. Resumo & obrigatório & $\begin{array}{l}\text { 20.Código de Caracteres dos } \\
\text { Metadados }\end{array}$ & condicional \\
\hline 10.Formato de Distribuiçåo & obrigatório & 21.Contato para Metadados & obrigatório \\
\hline $\begin{array}{l}\text { 11. Extensão Temporal } \mathrm{e} \\
\text { Altimétrica }\end{array}$ & opcional & 22.Data dos Metadados & obrigatório \\
\hline & & 23.Status & obrigatório \\
\hline
\end{tabular}


Para a implementação da INDE foi definido pelo Decreto 6.666/08 o Diretório Brasileiro de Dados Geoespaciais (DBDG) como um sistema de servidores de dados, distribuídos na internet, capaz de reunir eletronicamente produtores, gestores e usuários de dados geoespaciais, buscando o armazenamento, compartilhamento e acesso a esses dados e aos serviços relacionados.

O DBDG foi desenvolvido seguindo as normas e políticas definidas pelo e-PING (Padrões de Interoperabilidade de Governo Eletrônico). A arquitetura e-PING define um conjunto mínimo de premissas, políticas e especificações técnicas que regulamentam a utilização da Tecnologia de Informação e Comunicação (TIC) no governo federal brasileiro, para garantir a interoperabilidade na interação entre sistemas de diferentes instituições (BRASIL, 2010).

De acordo com esta arquitetura deve-se considerar as diferentes realidades tecnológicas dos atores envolvidos, assim o DBDG foi implementado considerando soluções para incorporar instituições com alta capacidade tecnológica e também as de menor capacidade. Em relação a dados espaciais, os padrões definidos pelo e-PING, e por consequência adotados no DBDG, são abertos e baseados nas definições do Open Geoespatial Consortium (OGC) (BRASIL, 2010).

No Plano de Ações da INDE (BRASIL, 2010) é definido o modelo conceitual do DBDG como mostra a Figura 6. É possível distinguir três camadas: a camada de aplicações, a camada intermediária e a camada dos servidores. A camada de aplicações é composta por navegadores web e aplicações situadas no domínio do cliente, como softwares de Sistemas de informações Geográficas, entre outros.

A camada intermediaria é composta pelo Portal SIG Brasil, o Módulo de Administração, o Catálogo Global de Metadados, o Catálogo de Servidores e o Servidor Web. O Portal SIG Brasil é responsável por possibilitar o acesso de forma simples aos recursos do DBDG, controlar o acesso as informações armazenadas, registrar os usuários, processar as requisições efetuadas pelos usuários e agregar metadados dos catálogos de servidores remotos.

O módulo de administração tem por objetivo oferecer ferramentas para manter e administrar o DBDG, acompanhando de forma precisa e constante a disponibilização de dados, elaborando estatísticas, cadastrando produtores de dados e gerenciando a interação entre os usuários e o Portal SIG Brasil. 


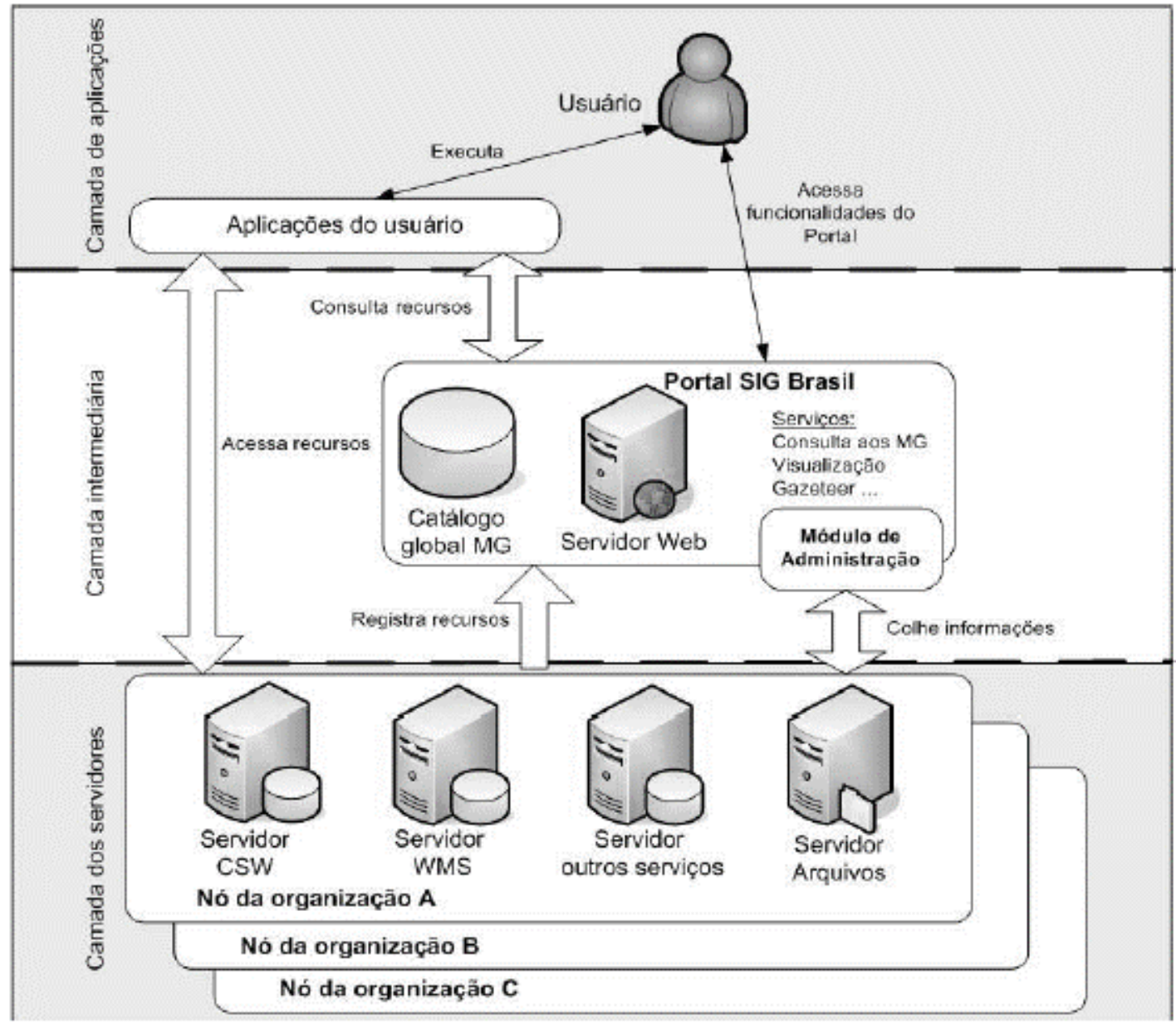

Figura 6 - Diagrama Conceitual do DBDG Fonte: Brasil, 2010

A camada de servidores é constituída por diversos nós, sendo cada nó um conjunto de servidores de responsabilidade de uma entidade provedora de dados geoespaciais. Assim o DBDG pode ser definido como uma rede de nós que interoperam por meio de interfaces baseadas em padrões abertos e se comunicam através da Internet (BRASIL, 2010).

Como nem todas as instituições brasileiras possuem infraestrutura suficiente para implementar e manter serviços para o funcionamento de uma estrutura totalmente baseada em Web Services existe um nó diferenciado que hospedam metadados e serviços geoespaciais destas instituições a partir de servidores administrados pelo IBGE.

Uma das maneiras mais efetivas para contribuir com o desenvolvimento da INDE é ingressar no DBDG como produtor ou provedor de dados. Uma instituição pode-se tornar um nó da INDE, para isto ela deve encaminhar uma solicitação a CONCAR, que irá avaliar o pedido e tomar as medidas necessárias para integrar esta nova instituição ao DBDG (INDE,2017).

\subsection{Pesquisas sobre IDE no Brasil e no Mundo}


Muitos países desenvolveram suas IDEs Nacionais, como a Austrália com a Australian Spatial Data Infrastructures (ASDI), os Estados Unidos com a National Spatial Data Infrastructure, a Holanda com a The National Geographic Informatio Infrastructure, a África do Sul com a National Spatial Information Framework (NSIF), a Colômbia com a Infraestructura Colombiana de Datos Espaciales (ICDE), a Indonésia com a Indonesian Spatial Data Infrastructure (ISDI), entre outros (ONAH, 2009).

Além das IDEs Nacionais, foram realizados estudos para o desenvolvimento de IDEs nas outras categorias hierárquicas, como IDEs estaduais (SOUZA, 2011; MARANHÃO, 2013) e IDEs locais desenvolvidas para questões ambientais e hidrológicas (DAVIS et al., 2009; NAKAMURA, 2010; LOPES, 2012; ORTIZ, 2012; CARVALHO, 2013). Oliveira (2014) propôs o desenvolvimento de uma IDE para a Copa do Mundo de 2014, a fim de disponibilizar dados espaciais para ajudar no translado entre as cidades sedes e os locais dos jogos.

Alguns estudos foram desenvolvidos com intuito de pesquisar sobre os componentes e tecnologias envolvidas na implementação de uma IDE. Davis (2017), Empraba (2012) e Fonseca (2016) desenvolveram trabalhos abordando a arquitetura e os aspectos computacionais empregados na criação de uma IDE. Oliveira et al. (2017) avaliou a utilização do modelo formal indicado pela Associação Internacional de Cartografia (ICA) para o desenvolvimento da estrutura de IDEs na formulação de uma IDE Corporativa. Através deste estudo, os autores constataram que o modelo se enquadrava às características da IDE estudada.

Neto (2010) desenvolveu um estudo buscando avaliar quais os aspectos deveriam ser considerados para integrar o Cadastro Nacional de Imóveis Rurais (CNIR) à INDE. Ele avaliou quais as mudanças necessárias à INDE e ao CNIR, para que este último se tornasse um dos nós da Infraestrutura de Dados Espaciais Nacional Brasileira.

Foram desenvolvidas pesquisas, na área da computação, a fim de extrair conhecimento a partir dos metadados disponibilizados em IDEs. Nestes estudos foram avaliados padrões em informações de metadados e aplicadas ferramentas de mineração de dados, como agrupamentos e classificações, buscando melhores formas para definir as buscas em catálogos de metadados (SILVA, 2010; MARTINS, 2012; MONTANARI, 2017).

A Informação Geográfica Voluntária (VGI) foi um tópico abordado no desenvolvimento de IDE (MIRANDA, 2011). Neste trabalho, o usuário atuava também como fornecedor de informações. Uma preocupação apontada nestes casos é a forma de avaliar a qualidade dos dados que são fornecidos voluntariamente, pois nem todos possuem conhecimento cartográfico prévio ou estão aptos a fornecer dados espaciais de confiança e qualidade.

A OpenStreetMap é uma iniciativa internacional que visa fornecer dados geográficos gratuitos e valoriza o conhecimento local. É uma comunidade constituída de colaboradores que mapeiam Revista Eletrônica: Tempo - Técnica - Território, V.9, N.2 (2018), 7:27 ISSN: 2177-4366 
voluntariamente estradas, ruas, edificações públicas e comércios de forma a elaborarem bases cartográficas. As informações mapeadas podem ser utilizadas livremente, pois os dados da OpenStreetMap são abertos (OpenStreetMap Foundation, 2017).

A OpenStreetMap conta com um geoportal (www.openstreetmap.org), onde apresenta as feições mapeadas, imagens de satélites e onde é possível realizar um cadastro para tornar-se um colaborador. Neste geoportal, existem ferramentas que permitem a edição e inserção de feições, as alterações feitas podem ser salvas, e após análise estas alterações se tornam efetivas e podem ser visualizadas e exportadas por qualquer usuário. A OpenStreetMap é um grande projeto, de nível internacional, que trabalha com coleta e distribuição de informações geográficas voluntárias.

Brito et al. (2014) abordou sobre a questão das IDEs Universitárias, mostrando o papel das universidades para o ensino e pesquisas relacionadas a IDE e também no desenvolvimento de IDEs. Neste trabalho ele apresenta que três universidades brasileiras já iniciaram o desenvolvimento de suas IDEs em 2012: a USP com o projeto IDE - USP, a UFBA com o projeto IDE - UFBA e a UFPR que criou o primeiro laboratório na América Latina que faz parte da Rede de Laboratórios Geoespacial Livres (Rede ICA-OSGEO Labs). A Rede ICA-OSGEO Labs é um acordo entre a ICA e a Fundação OSGEO que apoia o desenvolvimento de softwares livres da área geoespacial (BRITO et al., 2014).

Em 2013 foi criada a rede IDE-Acadêmica, esta rede contou com a participação de professores e pesquisadores da UFPR, UFRS, UFRRJ e UFPE (BRITO et al., 2014). Além destas iniciativas nacionais, algumas universidades internacionais também desenvolveram programas para criação de IDEs Universitárias. Sendo exemplos destes programas o projeto britânico GOGEO, o projeto australiano Australian Spatial Research Data Commons (ASRDC) e o projeto canadense do estado de Ontário Scholars GeoPortal (BRITO et al., 2014).

\subsection{Premissas para desenvolvimento de estudos futuros}

Com base em todos os estudos já desenvolvidos pode-se perceber que a Internet é uma ótima ferramenta para disponibilização de dados cartográficos, e que ainda há mais para se explorar. Existem muitos softwares destinados a publicação de dados espaciais na Internet, sendo vários de código livre e aberto, sem custos para aquisição e utilização. Logo, hoje a publicação de dados espaciais é possível e deve ser realizada. Estudos podem ser desenvolvidos para avaliar os softwares existentes, visando analisar a aplicação destes no desenvolvimento de IDEs.

Em relação ao desenvolvimento de IDE, a INDE está alinhada com as iniciativas de IDEs dos países desenvolvidos, em relação a adoção e criação de normas e políticas. No entanto, ainda é necessária uma maior divulgação e sensibilização para participação de outras camadas da pirâmide hierárquica (ORTIZ, 2012). 
O debate de IDEs deve ser incorporado a comunidade acadêmica, é necessário falar de IDE em aulas de graduação e pós-graduação, desenvolver mais projetos de pesquisa e extensão neste âmbito (BRITO et al., 2014). Além disto, as universidades devem se empenhar para desenvolverem uma IDE Universitária.

$\mathrm{Na}$ universidade em muitos projetos são produzidos dados e metadados que podem ser utilizados para alimentação de uma IDE sendo muitos destes estudos custeados por verbas públicas. Mas estes dados muitas vezes não são disponibilizados, se perdem nos laboratórios de pesquisa, e em alguns casos são levantados repetidas vezes para outros estudos.

Assim, a universidade é uma organização com potencial e necessidade de desenvolvimento de uma IDE. Uma IDE da Universidade poderá ser utilizada na área de ensino, como material didático; na área de pesquisa, potencializando a colaboração entre pesquisadores e evitando custos na geração duplicada de dados; e na área de extensão, tornando público a todos que necessitam os dados produzidos pela universidade (BRITO et al., 2014).

Uma IDE Universitária pode-se tornar também um nó da INDE, pois a academia é tratada como um ator importante na discussão das políticas, tecnologias, padrões e na capacitação de pessoas, e já começa a ser tratada também com um nó produtor de informação geográfica (BRITO et al., 2014).

Uma outra questão que pode ser abordada é o controle de qualidade dos dados disponibilizados em uma IDE. Uma IDE é formada por dados espaciais desenvolvidos por diferentes membros, empregando tecnologias distintas, assim a qualidade cartográfica em uma IDE varia de dado a dado. Se fazendo necessário a descrição detalhada de cada dado incorporado a IDE, para que o usuário seja capaz de definir se é possível a utilização daquele dado a sua necessidade (MARANHÃO, 2013).

A análise do controle de qualidade em IDEs é uma questão fundamental, não basta simplesmente disponibilizar os dados é necessário que os dados disponibilizados sejam confiáveis, para que estes possam ser reutilizados. Desta forma, existe a necessidade de desenvolvimento de estudos e pesquisas que busquem definir formas para se tratar a qualidade cartográfica das informações geográficas disponibilizadas em uma IDE.

\section{Considerações Finais}

A partir do entendimento das vantagens proporcionadas pela criação de uma IDE, pode-se perceber que além das esferas nacionais e estaduais, outros componentes da camada hierárquica devem atuar mais e se empenharem no desenvolvimento de infraestruturas para compartilharem as informações espaciais que produzem. 
Dentre estes componentes se destacam as universidades. Estas, além de produzirem e utilizarem informações espaciais, são os principais atores no desenvolvimento de pesquisas, para trazerem inovações e definições dos melhores padrões a serem utilizados na criação de IDEs.

Diante disto, os autores deste artigo desenvolveram a proposta de criação de uma IDE para a Universidade Federal de Viçosa (UFV). No desenvolvimento da IDE Universitária - UFV serão desenvolvidos estudos para definição de uma modelagem conceitual que contemple a realidade das universidades em relação a implementação de IDE e que seja compatível com os padrões da INDE.

Uma grande preocupação no desenvolvimento deste projeto é que a IDE a ser criada além de ser um projeto de pesquisa, seja algo realmente útil à Universidade e que existam mecanismos para garantir sua atualização e continuidade. Desta forma, está sendo dada uma atenção especial em relação as políticas necessárias para a implementação desta IDE. A proposta deste trabalho foi apresentada à Pró-Reitoria de Administração (PAD) da UFV, elucidando a importância de uma IDE para a universidade e quais as vantagens esta proporcionaria. A administração se mostrou interessada no desenvolvimento da infraestrutura e já está trabalhando no processo de institucionalização da IDE dentro da UFV.

Atualmente a UFV não possui uma base unificada de dados referentes aos campi universitários, o que acarreta perdas de tempo e recursos financeiros nos processos administrativos onde estes dados são essenciais. Desta maneira, existe a necessidade da administração de organizar as informações existentes e desenvolver mecanismos para torná-las mais acessíveis. Como forma de atrair a atenção da administração para o apoio no desenvolvimento e institucionalização da IDE para a UFV e mostrá-los de forma mais prática a importância de um IDE Acadêmica, inicialmente o foco desta IDE serão os dados de referência para a administração. Exemplos destes dados cartográficos de referência são as informações referentes aos logradouros, edificações, hidrografia, altimetria, uso e ocupação do solo, entre outros elementos do campus.

Outro objetivo deste projeto é tornar a IDE da UFV um nó próprio integrado à INDE. Esta integração aumentaria a abrangência de compartilhamento dos dados produzidos pela universidade e atenderia as obrigações dispostas no Decreto 6.666, quanto a divulgação dos dados cartográficos produzidos por entidades governamentais.

\section{Referências Bibliográficas}

BRASIL, Decreto $\mathbf{n}^{\circ}$ 6.666, de 27 de novembro 2008. 2008.

BRASIL, M. DO P. O. E G. Plano de Ação para implantação da INDE: Infraestrutura nacional de dados espaciais. Comissão Nacional de Cartografia(CONCAR), 2010. 
BRITO, P. L., SOUZA, F. A., CAMBOIM, S., GIANNOTTI, M. A., Primeiros passos para a implementação de uma IDE Universitária. In: SIMPÓSIO DE CIÊNCIAS GEODÉSICAS E TECNOLOGIAS DA GEOINFORMAÇÃO, 5, 2014, Recife - PE. Anais... Recife, 2014. p. 77-84.

BUDHATHOKI, N. R., BRUCE, B., NEDOVIC-BUDIC, Z. Reconceptualizing the role of the user of spatial data infrascture. GeoJournal, v. 72, p. 149-160, 2008.

CARVALHO, G. N., Proposta de tratamento e modelagem de dados espaciais para uso em Infraestrutura de Dados Espaciais - IDEs: Estudo de caso de Macrobentos para a área costeira da baixada santista. Dissertação de Mestrado (Apresentado ao programa de Mestrado em Engenharia). São Paulo: Escola Politécnica da Universidade de São Paulo, Departamento de Engenharia de Transportes. 2013

CRAGLIA, M., GOODCHILD, M. F., ANNONI, A., CAMARA, G., GOULD, M., KUHN, W., MARK, D., MASSER, I., MAGUIRE, D., LIANG, S., PARSONS, E., Next-Generation Digital Earth: A position paper from the Vespucci Initiative for the Advancement of Geographic Information Science. International Journal of Spatial Data Infrastructures Research, v. 3, p. 146-167, 2008.

DAVIS C. A., FONSECA, F. T., CÂMARA, G., Infraestruturas de Dados Espaciais na Integração entre Ciências e Comunidades para Promover a Sustentabilidade Ambiental. 2009. Disponível em: < http://homepages.dcc.ufmg.br/ clodoveu/files/100.40/AC049.\%202009\%20 Infraestruturas\%20de\%20dados\%20espaciais\%20na\%20integracao\%20entre\%20ciencia\%20e\%20c omunidades\%20para\%20promover\%20a\%20sustentabilidade\%20ambiental.pdf $>$. Acesso: 10 de maio de 2017.

EMBRAPA, Infraestrutura Nacional de Dados Espaciais - Aspectos Computacionais. Campinas, SP. 2012.

FONSECA, F. J. B. ; PEREIRA, L. V. S. ; LISBOA-FILHO, J. . Comparando sistemas WebGIS de exibição e processamento de informação geográfica no contexto da INDE. In: CONFERENCIA IBÉRICA DE SISTEMAS Y TECNOLOGÍAS DE INFORMACION (CISTI), 2016, Gran Canaria, Espanha. Actas... Gran Canaria: AISTI | ISEGI, 2016. v. 1. p. 250-255.

GSDI - GLOBAL SPATIAL DATA INFRASTRUCTURE ASSOCIATION, Developing spatial data infrastructures: The SDI Cookbook. United States, Janeiro de 2004.

INDE - INFRAESTRUTURA NACIONAL DE DADOS ESPACIAIS, Roteiro de Adesão a INDE.

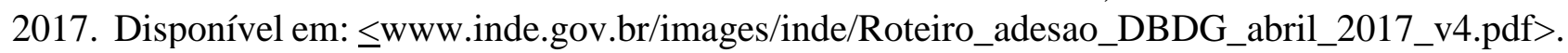
Acesso: 06 de julho de 2017.

JANSSEN, K., The influence of the PSI directive on open government data: Na overview of recente developments. Government Information Quartely, v. 28, p. 446-456, 2011.

LOPES, A. M., Integração de dados espaciais heterogêneos no domínio hidrológico utilizando Infraestrutura de Dados Espaciais. Dissertação de Mestrado (Apresentado ao programa de Mestrado em Ciência da Computação). Universidade Federal de Viçosa. 2012.

MARANHÃO, V. C., Modelagem e controle de qualidade de uma Infraestrutura de Dados Espaciais para o estado de Pernambuco. Dissertação de Mestrado (Apresentado ao programa de Mestrado em Ciências Geodésicas). Universidade Federal de Pernambuco. 2013.

MARTINS, E. S. ; LISBOA FILHO, J. ; RIBEIRO, M. H. F. ; REINALDO, F. . Mineração de Dados em Infraestruturas de Dados Espaciais usando Clusterização de Dados. In: CONFERENCIA LATINOAMERICANA DE INFORMÁTICA, 32, 2012, Medellín, Colômbia. Proceedings... Medellín: CLEI, 2012. p. 1-8 
MASSER, I. Report on A comparative analysis of NSDI's in Australia, Canada and the United States. GINIE: Geographic Information Network in Europe. Outubro de 2002.

MIRANDA, T. S. ; LISBOA FILHO, J. ; SOUZA, W. D. ; SILVA, O. C. ; DAVIS JUNIOR, C. A. . Volunteered geographic information in the context of local spatial data infrastructures. In: URBAN AND REGIONAL DATA MANAGEMENT (UDMS), 2011, Delft. Proceedings... Leiden: Taylor \& Francis, 2011. p. 123-138.

MONTANARI, M. V.; MOREIRA, A.; DIAS, V. E. C.; LOURENÇO, E.; LISBOA FILHO, J. Integrating a Metadata Editor with Hyperbolic Tree to Improve Data Access in Spatial Data Infrastructures. Communications in Computer and Information Science. 1ed.Cham, Switzerland: Springer International Publishing, 2017, v., p. 15-31

NAKAMURA, E. T., FILHO, A. P. Q., Infraestrutura de Dados Espaciais: Exemplo do Parque Estadual de Intervales - SP. Revista Brasileira de Cartografia, nº 645, p. 723-735, 2012.

NAKAMURA, E. T., Infraestrutura de Dados Espaciais em Unidade de Conservação: uma proposta para disseminação da informação geográfica do Parque Estadual de Intervales-SP. Dissertação de mestrado (Apresentada ao Programa de Pós Graduação em Geografia Física). São Paulo: Faculdade de Filosofia, Letras e Ciências Humanas, 2010.

NEDOVIC-BUDIE, Z., PINTO, J. K. Information sharing in na interorganizational GIS environment. Environment and Planning B: Planning and Design, v. 27, p. 455-474. 2000.

NETO, J. A. S., Análise da estruturação do Cadastro Nacional de Imóveis Rurais - CNIR com vistas à sua integração à Infraestrutura Nacional de Dados Espaciais - INDE. Dissertação de Mestrado (Apresentado ao programa de Mestrado em Ciências Geodésicas). Universidade Federal de Pernambuco. 2010.

OASIS - Organization for the Advancement of Structured Information Standards, Reference Model for Service Oriented Architecture 1.0. 2006.

OLIVEIRA, I. L.; CÂMARA, J. H. S.; TORRES, R. M.; LISBOA FILHO, J. Design of a Corporate SDI in Power Sector using a Formal Model. Infrastructures - Special Issue "Spatial Data Infrastructures", v.2, n. 4, p. 1-25, 2017.

OLIVEIRA, W. M. ; LISBOA FILHO, J. ; OLIVEIRA, A. P. . A spatial data infrastructure situationaware to the 2014 World Cup. In: INTERNATIONAL CONFERENCE ON COMPUTATIONAL SCIENCE AND ITS APPLICATIONS (ICCSA), 2012, Salvador. Proceedings... Berlin Heidelberg: Springer-Verlag LNCS 7333, 2012. p. 561-570.

ONAH, C. C., Spatial Data Infrastructures Model for Developing Countries: A case study of Nigeria. Dissertação de Mestrado (Apresentado ao programa de mestrado em Geospatial Technologies). Universitat Jaume. Março de 2009.

OpenStreetMap Foundation, About. 2017. Disponível em: $<$ https://wiki.osmfoundation.org/wiki /About>. Acesso em: 27 de Junho de 2017.

ORBA, R., STRUCH, J. C. M., SOUZA, M. S., Cartografia Ubíqua e Infraestrutura de Dados Colaborativa para Gestão de Eventos Geográficos. 2014. Disponível em: < http://docplayer.com.br/7072938-Cartografia-ubiqua-e-infraestrutura-de-dados-colaborativa-paragestao-de-eventos-geograficos.html>. Acesso: 15 de junho de 2017. 
ORTIZ, A. G. L., A Infraestrutura de Dados Espaciais do Intituto Chico Mendes de Conservação da Biodiversidade - Uma proposta de organização e compartilhamento. Dissertação de Mestrado (Apresentado ao programa de Mestrado em Geografia). Universidade de Brasília. 2012.

RAJABIFARD, A., BINNS, A, MASSER, I, WILIAMSON, I. P., The role of sub-national government and the private sector in future Spatial Data Infrasctructures. International Journal of Geographical Information Science, v. 20, p. 727-741, 2006.

RAJABIFARD, A., ESCOBAR, F., WILIAMSON, I. P., Hierarchical Spatial Reasoning Applied to Spatial Data Infrastructures. Cartography, v. 9, p. 41-50, 2000.

RAJABIFARD, A., FEENEY, M. F., WILIAMSON, I. P., Future directions for SDI development. International Journal of Applied Earth Observation and Geoinformation v. 4, p. 11-22, 2002.

RAJABIFARD, A., WILIAMSON, I. P., Development of a Virtual Australia Utilising na SDI Enabled Platform. In: PHARAOHS TO GEOINFORMATICS FIG WORKING WEEK 2005 AND GSDI-8, Cairo, Egito, 2005. Proceedings... Cairo, 2005. p. 1-11.

RAJABIFARD, A., WILIAMSON, I. P., Spatial Data Infrastructures: Concept, SDI Hierarchy and Future Directions. In: GEOMATICS'80 CONFERENCE, Tehran, Iran. 2001. Proceedings: Iran, 2001. p. 1-10.

ROCHA, D. S., Potencialidades do uso de um sistema WebGIS na pecuária de precisão: Estudo de caso Embrapa Pecuária Sul. Dissertação de Mestrado (Apresentado ao programa de Mestrado em Agricultura de Precisão). Universidade Federal de Santa Maria. 2014.

SILVA, O. C. ; FONTES, V. C. ; MIRANDA, T. S. ; LISBOA FILHO, J. ; OLIVEIRA, A. P. . Recuperação de Informação em Infraestruturas de Dados Espaciais usando Mecanismos de Representação do Conhecimento. In: WORKSHOP DE COMPUTAÇÃO APLICADA EM GOVERNO ELETRÔNICO (WCGE em conjunto com CSBC), 2010, Belo Horizonte. Anais... XXX Congresso da Sociedade Brasileira de Computação. Belo Horizonte: SBC, 2010. p. 634-647.

SOUZA, F. A., Avaliação da proposta de uma Infraestrutura de Dados Espaciais na Bahia e suas possíveis repercussões para estudos de impacto ambiental. Dissertação de Mestrado (Apresentado ao programa de Mestrado em Engenharia Ambiental Urbana). Universidade Federal da Bahia. 2011.

WARNEST, M. A collaboration model for National Spatial Data Infrastructure in Federated Countries. Tese de doutorado (Apresentada ao programa de doutorado em Philosophy). Departamento de Geomática da University of Melbourne. Fevereiro de 2005. 